\title{
The hydra-headed Mueller-Lyer effect: A theoretical puzzle
}

\author{
ALEXANDER W. PRESSEY and ALEXANDER E. WILSON \\ University of Manitoba, Winnipeg, Manitoba, Canada, R3T 2N2
}

\begin{abstract}
Three experiments were conducted to examine the effects of multiple fins on the magnitude of the Mueller-Lyer illusion. All studies showed that the multiple form of the elongation illusion was greater than the average of the components and that the multiple form of the shrinkage illusion was smaller than either of the components. The pattern of results failed to support either simple addition models or more complex averaging theories of illusions. A cross-attribute summation hypothesis and a filled space hypothesis were proposed as possible explanations for the results.
\end{abstract}

In the Mueller-Lyer configuration, shown in Figure 1, the distance between the apices of fins appears to shrink if the fins point inward and appears to elongate if the fins point outward. Such phenomenal distortions are called "visual illusions" and, of all the illusions that have been discovered, the MuellerLyer has been the most popular subject of both theory and research.

There now exist reliable data on the manner in which stimulus variables such as length, angle, color of fins, temporal and spatial separation between shaft and fins, and target-size affect the MuellerLyer illusion (Robinson, 1972). Moreover, these data have been used to develop and evaluate different theories of illusions (e.g., Pressey, 1972).

However, there is one stimulus variable that has not been investigated, and that is the number of fins that are placed at the ends of the shaft. Such a multiheaded target is shown in Figure 1C. This configuration is simply the sum of the targets shown in Figures $1 \mathrm{~A}$ and $\mathrm{IB}$.

The hydra-headed Mueller-Lyer is also important theoretically because it seems that simple additive models of illusions (e.g., Clavadetscher \& Anderson, 1977) would predict that the hydra-headed effect should be equal to the mean of the two separate effects, while more complex averaging theories (e.g.; Pressey \& Murray, 1976) suggest that the composite effect would lie in between (and not necessarily be the average) of the two simple effects. Therefore, the main purpose of the present study was to measure the effects of both an ingoing and an outgoing hydraheaded target and relate them to the effects produced by the simple targets.

This research was supported by the National Research Council of Canada (A0-177).
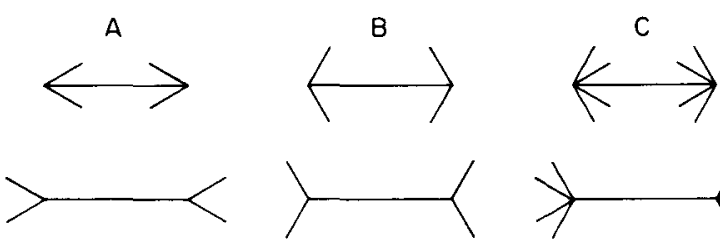

Figure 1. The ingoing (A), outgoing (B), and hydra-headed (C) forms of the Mueller-Lyer target.

\section{METHOD}

\section{Subjects}

Twenty-one men and women from introductory courses at the University of Manitoba participated in order to fulfill a course requirement. Only those with good vision, with or without glasses, were asked to participate.

\section{Materials}

Seven targets, the six shown in Figure 1 and a control line, were drawn with black ink on separate $27.8 \times 21.5 \mathrm{~cm}$ sheets of white paper. The lines between the fins, and the control lines, were $50 \mathrm{~mm}$ long and were oriental parallel to the narrow edge of the sheet. All standard lines were centered $9.1 \mathrm{~cm}$ below the top of the sheet.

The interior angle formed by the fins was either 60 or $120 \mathrm{deg}$. The hydra-head target contained both sets of fins. The lines forming the fins were $15 \mathrm{~mm}$ long and all lines in the target were $.5 \mathrm{~mm}$ wide.

Since the method of production was employed, no comparison line was actually presented. Instead, a small, black dot which served as a starting point was drawn in one of two positions. The "left" position was $10 \mathrm{~cm}$ below and $8 \mathrm{~mm}$ to the left of the left edge of the standard line and the "right" position was $10 \mathrm{~cm}$ below and $8 \mathrm{~mm}$ to the right of the left edge of the standard line.

All targets were duplicated by offset printing and presented on a white target holder that was $36.8 \mathrm{~cm}$ wide and $39.5 \mathrm{~cm}$ high. The face of the holder was tilted backwards $20 \mathrm{deg}$ in order to ensure that the entire target was perpendicular to the line of sight. A chinrest was placed in front of the holder so that the distance between the subject's eyes and the face of the frame was approximately $41 \mathrm{~cm}$.

\section{Procedure}

Each subject was seated in front of the apparatus, where he 
placed his chin on the rest and was shown a sample target. He was told that his task was to start at the dot and draw a line that appeared equal to the standard horizontal line. (The line was pointed out to the subject.) He was asked not to concern himself with the straightness of the line, since the experimenters were interested primarily in the length of the produced line.

Each subject was tested twice on all seven targets given in a unique random order. The two trials varied in the starting position of the dot. This position was counterbalanced within and between subjects.

\section{RESULTS AND DISCUSSION}

Measurements of the produced line were made with a transparent millimeter scale, and these measurements were accurate to within $.5 \mathrm{~mm}$. The scores from the two trials were averaged to provide one score for each of the six targets. Then scores from the control target were subtracted from the scores on each of the remaining targets to provide measures of illusion. The results are plotted in the first panel in Figure 2.

A within-subjects analysis of variance showed that the type of target (ingoing fins vs. outgoing fins) was significant $(F=7.26, \mathrm{df}=1,20, \mathrm{p}<.02)$ and the angle of fins was significant $(F=4.09$, $\mathrm{df}=2,40, \mathrm{p}<.05$ ).

There are two puzzling features about the results. Only the data from the outgoing target (expansion illusion) seemed to support the idea that the MuellerLyer hydra was the average of its parts. The ingoing hydra produced a substantially smaller illusion than would be expected on the averaging hypothesis. The second puzzling feature was that angle of fins appeared to have no effect on the degree of illusion produced by the inward-pointing fins; that is, the fins with an interior angle of $120 \mathrm{deg}$ produced an illusion as large as the fins with a 60-deg angle.

Because of these puzzling features, it was decided to replicate the experiment in the hope that the results

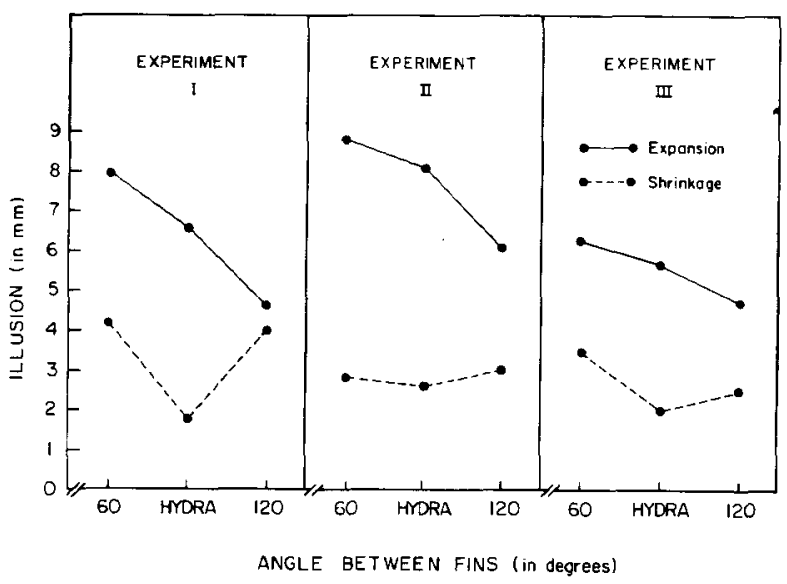

Figure 2. The effect of angle and number of fins on the ingoing and outgoing forms of the Mueller-Lyer illusion. would be clarified. Thirty-four subjects were chosen from the same population as in the first experiment and subjected to the same test and procedure. The results are shown in the second panel of Figure 2. Analysis of variance showed that the type of target was significant $(\mathrm{F}=29.3, \mathrm{df}=1,33, \mathrm{p}<.01)$, the angle of fins was significant $(\mathrm{F}=4.02$, $\mathrm{df}=2,66$, $\mathrm{p}<.05$ ), and the Target by Angle interaction was significant $(F=5.73, \mathrm{df}=2,66, \mathrm{p}<.01)$.

It is clear from Figure 2 that the patterns of results from the second study were very similar to those of the first except that it now appeared that neither the ingoing nor the outgoing Mueller-Lyer hydra was the average of its components. Indeed, it seemed that the proper generalization was that the outgoing hydra was greater than the average of its components and that the ingoing hydra was less than the average of its components.

However, because the trends were so unexpected, it was decided to provide a final replication. Ninetythree subjects from the same population used in the previous two studies were tested. Group testing was employed in a manner very similar to that described by Pressey and Sweeney (1972). The targets were identical to those used in the previous experiments. The results are shown in the third panel of Figure 2. Once again analysis of variance showed that the variables of type of target, angle of fins, and the interaction of target and angle were all significant beyond the .05 level of confidence. Thus, we can conclude with confidence that the hydra-headed Mueller-Lyer illusion is not equal to the average of its components.

How can we explain the results shown in Figure 2? They do not support simple additive models (Clavedetscher \& Anderson, 1977) or more complex averaging theories (e.g., Pressey \& Murray, 1976), which suggests that the composite effect would be equal to the average or lie in between the simple effects, respectively. In fact, the ingoing form of the hydra-headed target consistently produced a smaller effect than either of the two more simple versions of the ingoing figure. The most obvious interpretation is that each hydra-headed target produces an independent elongation effect which adds to the elongation illusion and subtracts from the shrinkage illusion. If this hypothesis is accepted, there are two possibilities for such an elongation effect. The first is a numerosity hypothesis. It may be argued that the hydra-headed target just has more "things" in it and that this greater number becomes translated into greater length. This hypothesis can be labeled the "cross-attribute summation hypothesis."

A second possibility for the general elongation is that a filled space illusion is involved. If one looks at the hydra-headed target, one can see that the space between the distal fins in both forms of the target is filled with the fins of the remaining target. 
Now, if it is true, for example, that the MuellerLyer illusion is due to assimilation of the standard length to lengths produced by the fins (Pressey, 1971), and if it is true that the amount of assimilation is directly related to the length of the contextual distance (Pressey, 1972), then in the outgoing hydra target the contextual distance will be larger and thus produce a greater elongation illusion. On the other hand, the filled space effect will also lengthen the apparent size of the contextual distance in the ingoing form of the illusion. But, since these distances were initially smaller than the standard (thus producing shrinkage), the lengthening of the contextual distances reduces the illusion.

Regardless of which interpretation is correct, the results of this study reinforce the Gestalt dictum that the whole is different from the sum of its parts. And it appears that no theory of illusions at present adequately upholds that dictum.

\section{REFERENCES}

Clavadetscher, J. E., \& Anderson, N. H. Comparative judgment: Tests of two theories using the Baldwin figure. Journal of Experimental Psychology: Human Perception and Performance, 1977, 3, 119-135.

Pressey, A. W. An extension of assimilation theory to illusions of size, area, and direction. Perception \& Psychophysics, 1971, 9, 172-176.

Pressey, A. W. The assimilation theory of geometric illusions: An additional postulate. Perception \& Psychophysics, 1972, 11. 28-30.

Pressey, A. W., \& Murray, R. Further developments in the assimilation theory of geometric illusions: The adjacency principle. Perception \& Psychophysics, 1976, 19, 536-544.

Pressey, A. W., \& Sweeney, $O$. Acute angles and the Poggendorff illusion. Quarterly Journal of Experimental Psychology, 1972, 24, 169.174.

Rosinson, J. O. The psychology of visual illusion. London: Hutchinson, 1972.

(Received for publication March 21, 1977; revision accepted July $1,1977$. 